\title{
Uma análise orientada a dados para avaliar o impacto da gamificação de um juiz on-line no desempenho de estudantes
}

\author{
Daniel L. Z. Filho ${ }^{1}$, Elaine H. T. Oliveira ${ }^{1}$, Leandro S. G. Carvalho ${ }^{1}$, Marcela Pessoa ${ }^{1}$, \\ Filipe Dwan Pereira ${ }^{2}$, David B. F. Oliveira ${ }^{1}$ \\ ${ }^{1}$ Instituto de Computação - Universidade Federal Amazonas (UFAM) \\ ${ }^{2}$ Departamento de Ciência da Computação - Universidade Federal de Roraima (UFRR) \\ \{dlzf, david, galvao, elaine, msppessoa\}@icomp.ufam.edu.br, filipe.dwan@ufrr.br
}

\begin{abstract}
The use of gamification in educational settings can have positive, neutral or even negative effects. Therefore, it is essential to assess the impact of gamification on student performance. Recent studies point to the use of analysis directed at student data to assess gamification instead of questionnaires that are poorly scalable and may present biases that depend on many factors. In fact, this article presents a study on the effects of a structural gamification platform integrated to an online judge with its own IDE (Integrated Development Environment), for classes in the Introduction to Computer Programming (IPC) discipline. The study carried out on 9 classes analyzes the correlations between the time that students spend in the programming environment and on the gamification platform, and their respective performances based on their final grades. As a result, a positive correlation was observed between practice time and performance, which was shown to be positively correlated with gamification.
\end{abstract}

Resumo. O uso da gamificação em ambientes educacionais pode ter efeitos positivos, neutros ou até negativos. Por isso, é essencial avaliar o impacto da gamificação no desempenho dos alunos. Estudos recentes apontam para o uso de análise dirigida aos dados dos estudantes para avaliar a gamificação ao invés de questionários que são pouco escaláveis e podem apresentar vieses que dependem de muitos fatores. Seguindo essa linha, este artigo apresenta um estudo sobre os efeitos de uma plataforma de gamificação estrutural integrada a um juiz on-line com IDE (Integrated Development Enviroment) próprio, para turmas da disciplina de Introdução à Programação de Computadores (IPC). $O$ estudo realizado sobre 9 turmas analisa as correlações entre o tempo que alunos passam no ambiente de programação e na plataforma de gamificação, $e$ seus respectivos desempenhos baseados em suas notas finais. Como resultados, observou-se uma correlação positiva entre o tempo de prática e o desempenho que mostrou-se correlacionada positivamente com a gamificação.

\section{Introdução}

A gamificação tem atraído a atenção de muitos pesquisadores da área de educação, como uma alternativa para aumentar a motivação e o engajamento dos alunos, de forma a melhorar o desempenho escolar [Sailer and Homner 2020]. No entanto, atualmente, não há diretrizes práticas sobre como conduzir sistematicamente a integração da gamificação em diversas experiências educacionais [Dichev and Dicheva 2017]. Essa falta de diretrizes pode ocasionar diferentes resultados, positivos e negativos, após a utilização da gamificação no contexto educacional [Toda et al. 2017]. 
Grande parte das pesquisas sobre os efeitos da gamificação na educação é avaliada ou examinada por meio de abordagens qualitativas, mediante o uso de pesquisas e questionários [Ogawa et al. 2016]. No entanto, à medida em que aumenta o interesse da academia e das instituições de ensino por sistemas educacionais gamificados, aumenta também a importância de se desenvolver formas cada vez mais precisas de se avaliar tais sistemas. Apesar da importância das ferramentas baseadas em respostas do usuário, neste contexto, elas podem gerar viés. Por exemplo, i) fazer perguntas sobre o nível de motivação do aluno enquanto ele está resolvendo uma atividade pode distraí-lo; ou ii) aplicar um questionário ao final da atividade pode levar a conclusões erradas, uma vez que o engajamento e a motivação são estados afetivos que possuem gatilhos específicos e podem não durar muito tempo [Pereira et al. 2020].

Estudos recentes [Meder et al. 2017, Pereira et al. 2020] apontam para a efetividade da avaliação da gamificação através de dados de interação dos alunos com os ambientes gamificados, mediante o uso de técnicas de mineração de dados e análises estatísticas. Essas análises indicam como os alunos entendem e utilizam o sistema podendo revelar pontos positivos e negativos da abordagem de gamificação usada. Para representar o comportamento dos alunos, são utilizadas as informações geradas em suas interações com os sistemas, sem a necessidade de fazer perguntas aos alunos, tornando a avaliação menos invasiva e mais escalável.

Desta forma, o intuito deste trabalho é analisar o impacto do uso de uma plataforma de gamificação no desempenho dos alunos da disciplina Introdução à Programação de Computadores (IPC), considerando os aspectos comportamentais desses alunos ao utilizar uma plataforma gamificada integrada a um juiz on-line. A plataforma gamificada foi integrada ao sistema juiz on-line CodeBench, que é utilizado na Universidade Federal do Amazonas, por professores e alunos, como ferramenta de apoio nas turmas de IPC. O sistema foi usado por 9 turmas, de cursos de Ciências Exatas e Engenharias, no primeiro semestre de 2019.

Portanto, este trabalho levantou à seguinte questão de pesquisa Q1: Em relação ao desempenho de alunos de turmas de introdução à programação, qual a influência/impacto da gamificação utilizada em um juiz on-line?

\section{Trabalhos Relacionados}

Muitos estudos [Meder et al. 2017, Pereira et al. 2020, Toda et al. 2017] afirmam que ainda não está claro como se deve planejar um ambiente gamificado de sucesso. Faltam teorias sobre as relações entre os elementos do design do jogo, a motivação, o contexto do domínio e o comportamento do usuário [Toda et al. 2017]. Segundo [Meder et al. 2017], para expandir o conhecimento nessa área, é necessário coletar e analisar dados de comportamento do usuário e dados de interações com elementos gamificados, coletados em estudos do mundo real. Nesse sentido, [Meder et al. 2017] definiram o tópico "Design de Gamificação Orientada a Dados" como: "automação do processo de design de gamificação usando abordagens de mineração de dados para aplicar elementos de design de jogos personalizados para cada indivíduo".

Assim sendo, [Stuart et al. 2020] estudaram os dados de uso de 258 alunos com cerca de 13 anos que usaram um ambiente de aprendizado gamificado como parte de suas aulas de matemática. Os autores mostraram um modelo de aprendizado para adaptar a gamificação baseada no perfil do jogador e no perfil de motivação do aluno. Porém, essa adaptação obteve efeitos diversos na motivação e no engajamento, isto é, segundo os au- 
tores, as influências medidas para cada elemento do jogo podem ser diferentes para outros alunos. Para ilustrar, estudantes mais novos podem ser mais receptivos à diversão induzida por elementos de jogo, enquanto que alunos mais velhos, ou menos fluentes em tecnologia, podem ser menos receptivos. Ainda, os autores apontam que é necessário analisar os impactos da gamificação em contextos educacionais diferentes, como por exemplo: idade, classe social, cursos (em caso de especializações, graduações e outros).

Já [Pessoa et al. 2019] analisaram os impactos de um juiz on-line integrado a uma plataforma de gamificação utilizada por turmas de programação. Baseados no tempo de utilização do sistema juiz on-line, notas finais e questionários, os resultados indicaram boa receptividade pelos alunos, aumento no tempo que utilizaram o juiz on-line e na quantidade de alunos com média final igual a 10,0. Entretanto, não houve aumento na taxa de aprovação dos alunos. O estudo de [Pessoa et al. 2019], porém, não analisou o tempo que o aluno passou utilizando a plataforma de gamificação, tampouco, foi realizada uma análise estatística para avaliar como o tempo de utilização do juiz on-line e da plataforma gamificada podem explicar a variação das notas finais dos alunos.

Utilizando técnicas de aprendizagem de máquina com elementos de gamificação como parâmetros, [Pereira et al. 2020] obtiveram $89 \%$ de precisão para prever se um estudante de ciência da computação seria ou não aprovado em um curso de programação. Os estudantes utilizaram o sistema juiz on-line URI, gamificado com ranqueamento de pontuações baseadas no desempenho e elementos de renovação. O estudo também apontou que esses elementos de gamificação incentivaram a competição e colaboração e que a análise de dados através dos algoritmos de predição, utilizando a gamificação, podem ser úteis para os professores acompanharem o desempenho dos estudantes e para melhor entender o relacionamento dos elementos de gamificação com o desempenho dos discentes.

Por fim, destaca-se que o presente trabalho realiza uma análise estatística de dados temporais coletados a partir da interação do estudante com um ambiente gamificado em turmas de programação. Como foi apontado pelos trabalhos citados nesta seção, uma avaliação do impacto da gamificação é desejável para expandir o conhecimento desse campo. Especificamente, é importante analisar estatisticamente os impactos da gamificação no comportamento dos alunos. O atual estado da arte carece de análises voltadas para avaliar o comportamento dos usuários mediante o tempo de uso do sistema. Coletar e analisar essas informações permitirá entender melhor como aplicar a gamificação de maneira tanto geral como adaptada aos perfis dos alunos.

\section{Contextualização}

\subsection{Juízes on-line}

Juízes on-line são sistemas que disponibilizam exercícios de programação e recebem códigos-fonte, que são corrigidos automaticamente utilizando casos de teste précadastrados. São comumente empregados como ferramenta de apoio pedagógico, pois diminuem a carga de trabalho do professor e oferecem feedback instantâneo para os alunos [Dwan et al. 2017].

O juiz on-line usado no presente estudo é um sistema que se encaixa na categoria de juízes educacionais. O sistema possui um IDE (Integrated Development Enviroment, em português - Ambiente de Desenvolvimento Integrado) próprio com editor de códigos, compilador e console de teste. Os professores elaboram as listas de exercícios e determinam o intervalo de tempo em que os estudantes podem acessar e responder essas listas. 
Assim, na área da computação, sistemas como o juiz on-line oferecem para os professores um maior controle sobre os códigos desenvolvidos pela classe e, para os alunos, serve de ambiente de resolução de exercícios com correção automática [Pereira et al. 2019].

\subsection{Gamificação}

$\mathrm{Na}$ área de educação, o método da gamificação emprega elementos adequados dos jogos para motivar o engajamento dos alunos nos assuntos lecionados [Klock et al. 2018]. Baseado nessa ideia, a plataforma de gamificação CodePlay, utilizada neste trabalho, disponibiliza um jogo multiplayer com elementos característicos de um jogo de RPG: narrativa imersiva, personagens, criaturas, ambientes e mistérios. Dentro do jogo, o aluno se vê como um dos personagens no mundo fictício onde o jogo se passa e pode caminhar pelos mapas, interagir com os demais alunos, vencer inimigos, investigar as histórias e explorar ambientes [Pessoa et al. 2019].

A plataforma não influencia no conteúdo da disciplina, nem é de uso obrigatório para os alunos. Entretanto, quando o aluno resolve corretamente as listas de exercícios disponibilizadas no juiz on-line pelo professor, recebe recompensas dentro do jogo que lhe permitem avançar na história, desbloquear novos itens e novas interações com os ambientes dentro jogo. Dessa forma, o jogo recompensa aqueles que se dedicam à resolução de exercícios [Pessoa et al. 2019].

\section{Metodologia}

O presente trabalho baseia-se na coleta de informações geradas por meio da interação dos alunos com o sistema juiz on-line e com a plataforma de gamificação. O método consiste em dois passos: i) coleta dos dados; e ii) engenharia de atributos. Cada um deles será discutido a seguir.

\subsection{Coleta de dados e Extração de Atributos}

A coleta de dados é a primeira etapa da metodologia. Consiste na definição de um conjunto de atributos possivelmente importantes para o estudo e na extração dos valores desses atributos a partir da base de dados do juiz on-line ${ }^{1}$. Tais dados foram extraídos de uma coleção de 9 turmas da disciplina de IPC, totalizando 422 alunos matriculados dos cursos de Ciências Exatas e Engenharias da instituição, no primeiro semestre de 2019.

Neste estudo, optou-se por não usar questionários, uma vez que, buscou-se identificar os impactos da gamificação através da análise de correlações entre os dados oriundos do uso da plataforma de gamificação e o desempenho dos alunos observados. Para isso, foi usada a nota final para analisar o desempenho geral do aluno. Para representar o comportamento, foi usado o tempo durante o qual os estudantes passaram interagindo com o sistema, tanto com o IDE quanto com a plataforma de gamificação. Os dados foram armazenados durante todo o semestre em suas interações com o sistema, incluindo o tempo de uso em laboratórios de programação durante a aula. É importante ressaltar que o estudo não leva em consideração as atividades e o comportamento dos alunos fora do sistema. Dessa forma, foram definidos 3 atributos:

- Tempo de uso do IDE: o tempo que o aluno passou dentro do ambiente de programação do juiz on-line;

\footnotetext{
${ }^{1} \mathrm{http} / / /$ codebench.icomp.ufam.edu.br/dataset/
} 
IX Congresso Brasileiro de Informática na Educação (CBIE 2020)

Anais do XXXI Simpósio Brasileiro de Informática na Educação (SBIE 2020)

- Tempo de jogo: o tempo que o aluno passou dentro do jogo na plataforma de gamificação;

- Nota final: a nota final na disciplina, sendo a média dos trabalhos e avaliações.

Para calcular o tempo de uso, tanto no IDE quanto no jogo, é importante identificar os momentos em que o estudante está de fato interagindo com cada um dos ambientes do sistema. Isso foi feito através da base de dados do juiz on-line, que registra todas as ações que os alunos desempenham em ambos os sistemas. No IDE, por exemplo, é possível identificar quando o aluno está usando a aplicação através de cliques de mouse e uso do teclado. No jogo, é possível identificar a atividade por meio dos movimentos do usuário na plataforma de gamificação, além das interações que o aluno realiza com os elementos do jogo, como por exemplo, selecionar uma arma para controlá-la.

\subsection{Engenharia de atributos}

A nota final da disciplina é a média dos laboratórios de codificação e das provas. As provas são realizadas em sala de aula através do IDE, sem consulta e com tempo determinado. Entretanto, o tempo acumulado nos sistemas durante as provas não foi contabilizado, pois isso foge do intuito do trabalho que é analisar a motivação dos alunos em utilizar os sistemas livremente.

Os tempos de uso dos sistemas foram calculados através da soma dos intervalos de tempo em que os usuários interagem com cada sistema. Para cada aluno foram montadas linhas em ordem cronológica: uma linha das interações com a IDE e uma linha das interações com o jogo. O tempo de uso acumulado foi obtido somando os intervalos de tempo entre as interações. No entanto, foi observado que é comum que os alunos deixem de interagir com os sistemas durante breves intervalos de tempo, mesmo que tais ambientes estejam abertos em seus navegadores. Isso pode ocorrer, por exemplo, quando o aluno precisa conversar com alguém ou fazer um breve lanche.

Para evitar que o tempo em que o aluno passou inativo seja considerado nas análises, foi estabelecido um limiar de 60 segundos de tolerância para esses intervalos de uso, conforme adotado por Dwan et al. (2017). Dessa forma, sempre que o intervalo de tempo entre a interação atual e a última antes dessa era maior que 60 segundos, era considerado que o aluno não estava interagindo com o sistema e esse intervalo de tempo era descartado da análise.

\section{Resultados e discussões}

Para responder a questão de pesquisa Q1 (Em relação ao desempenho de alunos de turmas de introdução à programação, qual a influência/impacto da gamificação utilizada em um juiz on-line), foram formuladas duas hipóteses nulas:

- H1: A correlação entre o tempo de jogo e a nota do aluno não é estatisticamente significante.

- H2: A correlação entre o tempo de jogo e o tempo de IDE não é estatisticamente significante.

Primeiramente, foram analisados os conjuntos de dados apresentados na Figura 1. É possível observar que as 3 variáveis estudadas seguem distribuições assimétricas à direita, logo, os dados dos estudantes se concentram em geral (moda e mediana) nos valores mais baixos. Além disso, as variáveis apresentam Coeficientes 
de Variação $\left(C V=\frac{\text { Std.Dev. }}{\text { Mean }} 100\right)$ altos $\left(C V_{\text {nota }}=93,56 \%, C V_{\text {tempoDeIde }}=79,81 \%\right.$ e $C V_{\text {tempoDeJogo }}=84,01 \%$ ), o que indica que as notas e o tempo de interação dos alunos com o IDE e com o ambiente gamificado podem variar bastante, implicando em uma heterogeneidade no comportamento dos alunos. No mais, observa-se que as três variáveis possuem distribuições similares e que elas tendem a variar juntas, mas não necessariamente na mesma taxa, o que sugere uma correlação monotônica ${ }^{2}$ entre elas. Observa-se ainda, baixo desempenho $($ Mean $=3,31)$ em relação às notas dos alunos. De fato, o histograma das notas mostra que muitos estudantes $(N \approx 90)$ atingiram valores tendendo a zero (moda da distribuição), o que será discutido mais à frente.
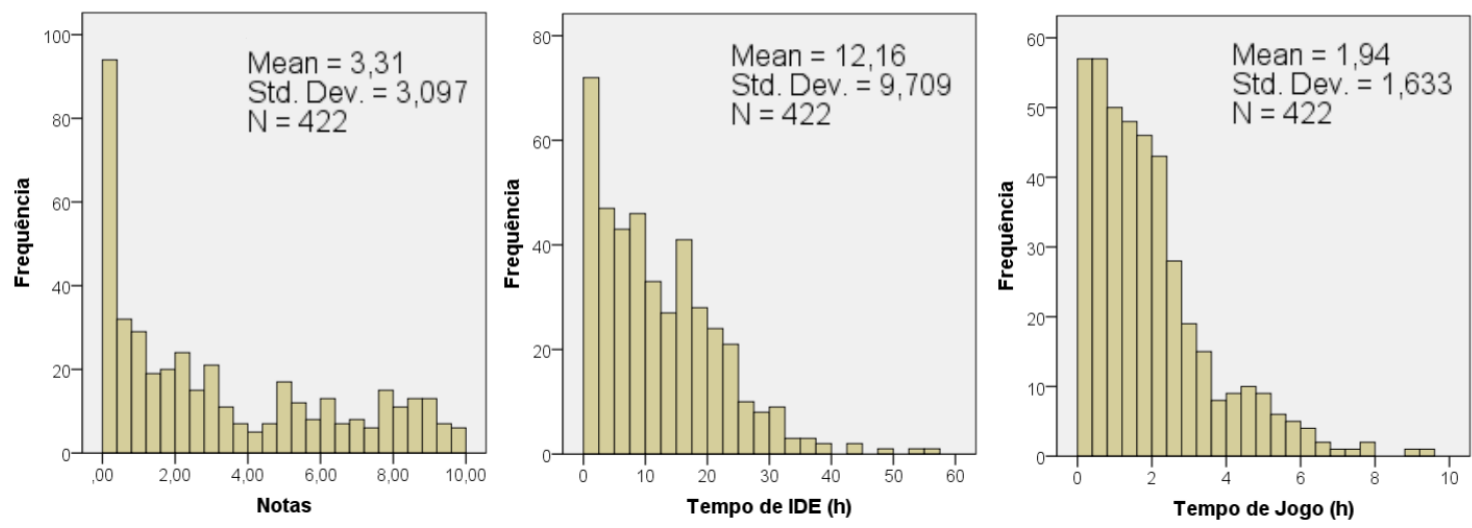

Figura 1. Distribuição das variáveis Notas, Tempo de IDE e Tempo de Jogo

Para um melhor entendimento das relações entre as variáveis, a Figura 2 mostra a dispersão das notas finais dos alunos (pontos em vermelho) e os tempos acumulados (em azul, o tempo de IDE e em amarelo, o tempo de jogo). É possível observar no gráfico que os conjuntos não possuem relações lineares, e que a parcela com menor nota também é, aparentemente, a que menos acumulou tempo no sistema. Isso resultou em uma inclinação acentuada na curva de crescimento dos tempos acumulados em relação às notas.

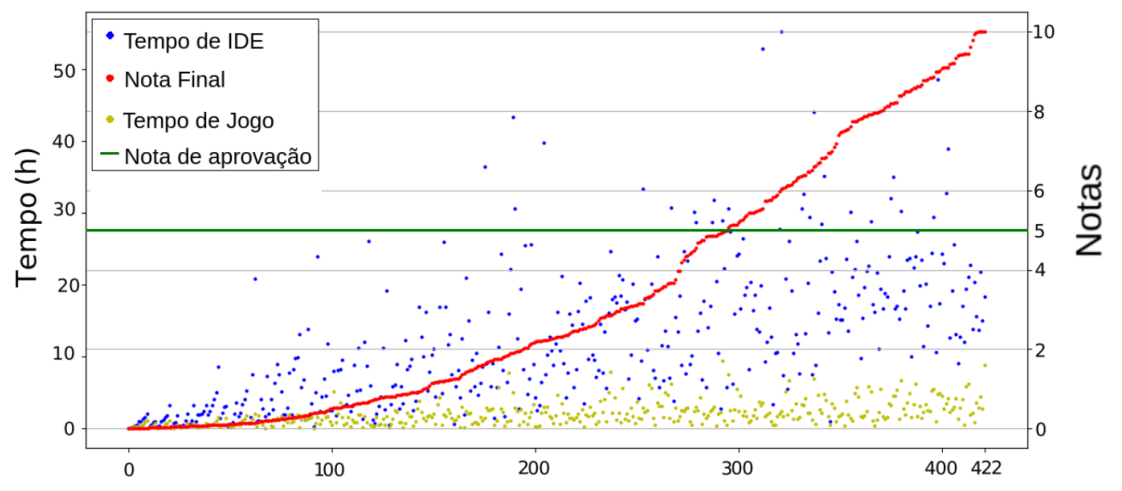

Figura 2. Cada posição no eixo $X$ representa um aluno com nota em vermelho, tempo de IDE em azul e de jogo em amarelo. $O$ eixo $Y$ da esquerda representa 0 tempo em horas e o eixo $\mathrm{Y}$ da direita representa as notas de $\mathbf{0 , 0}$ a 10,0.

Diante das inspeções visuais a partir da Figura 1 e da Figura 2, nota-se que o coeficiente de correlação de Pearson não é indicado para mensurar a correlação entre as variáveis de tempo e as notas, já que esse coeficiente assume uma relação de linearidade entre os dados, que, como observado, não é verdadeiro. Portanto, para avaliar as duas hipóteses, foi utilizado o coeficiente de correlação de Spearman (Tabela 1). Essa

\footnotetext{
${ }^{2}$ As variáveis tendem a mudar juntas mas não necessariamente a uma taxa constante.
} 
IX Congresso Brasileiro de Informática na Educação (CBIE 2020)

Anais do XXXI Simpósio Brasileiro de Informática na Educação (SBIE 2020)

correlação avalia a relação monotônica entre duas variáveis contínuas ou ordinais. O coeficiente de correlação de Spearman baseia-se nos valores classificados de cada variável.

Tabela 1. Correlação $\rho$ de Spearman entre as variáveis Notas, Tempo de IDE e Tempo de Jogo.

\begin{tabular}{|c|c|c|c|c|}
\hline & & Nota & $\begin{array}{r}\text { Tempo de } \\
\text { IDE }\end{array}$ & $\begin{array}{r}\text { Tempo de } \\
\text { Jogo }\end{array}$ \\
\hline \multirow{3}{*}{ Notas } & Coeficiente de correlação & 1,000 & $0,739 *$ & $0,604 *$ \\
\hline & Sig. (bicaudal) & . & 0,000 & 0,000 \\
\hline & $\mathrm{N}$ & 422 & 422 & 422 \\
\hline \multirow{3}{*}{ Tempo de IDE } & Coeficiente de correlação & $0,739 * *$ & 1,000 & $0,731^{*}$ \\
\hline & Sig. (bicaudal) & 0,000 & . & 0,000 \\
\hline & $\mathrm{N}$ & 422 & 422 & 422 \\
\hline \multirow{3}{*}{ Tempo de Jogo } & Coeficiente de correlação & $0,604 *$ & $0,731^{*}$ & 1,000 \\
\hline & Sig. (bicaudal) & 0,000 & 0,000 & \\
\hline & $\mathrm{N}$ & 422 & 422 & 422 \\
\hline
\end{tabular}

* A correlação é significativa no nível 0,01 (bicaudal).

A Tabela 1 apresenta a correlação de Spearman entre as variáveis Nota, Tempo de $I D E$ e Tempo de Jogo. Há indícios de uma forte correlação entre o tempo que o estudante passa programando no IDE e sua nota final, o que é esperado, já que o aluno que dedica mais tempo praticando tende a obter melhores resultados nas avaliações. Observa-se, ainda, que a correlação entre nota e tempo de jogo também é considerada forte. De fato, há significância estatística $(p<0,05)$ na correlação entre essas variáveis.

Além disso, nota-se uma correlação forte entre o tempo de IDE e o tempo de jogo $\left(\rho_{S}=0,731\right)$. Tal correlação, também, possui significância estatística $(p-$ value $<0.05)$. Isso indica que alunos com maiores notas passaram mais tempo no juiz on-line resolvendo exercícios e utilizando a gamificação, o que sugere uma relação da gamificação com comportamentos positivos (passar mais tempo resolvendo exercícios) e um desempenho alto, já que a correlação é positiva e, portanto, as variáveis tendem a crescer juntas.

Por outro lado, parte dos alunos desistia rápido das resoluções dos exercícios, acessando poucas vezes o ambiente de programação e o jogo, consequentemente acumulando pouco tempo de uso no sistema e obtendo notas muito baixas (pode ser observado na região entre 0 e 100 demarcados pelo eixo x na Figura 2). No gráfico da Figura 2, observa-se que uma parcela dos alunos acumulou menos de 5 horas no IDE, o que representa pouco tempo, considerando que só a carga horária prática em sala da disciplina é de 30 horas. Também, verifica-se que todos eles ficaram com notas finais abaixo de 1,0. Em contrapartida, os que se esforçaram mais acumularam relativamente muito mais tempo.

Além disso, para analisar segregadamente a relação do desempenho do aluno com o tempo de uso do IDE e do jogo, foram separados os dados dos estudantes reprovados e aprovados na disciplina de IPC. A Figura 3 mostra as distribuições e os gráficos de dispersão do tempo de IDE e do tempo de jogo dos alunos separados pela situação final.

As distribuições apresentadas na Figura 3, tanto de tempo de IDE como de tempo de jogo, se concentram em valores maiores de tempo de uso para o grupo de alunos aprovados. Para validar se essa tendência é estatisticamente significante, foi aplicado o 

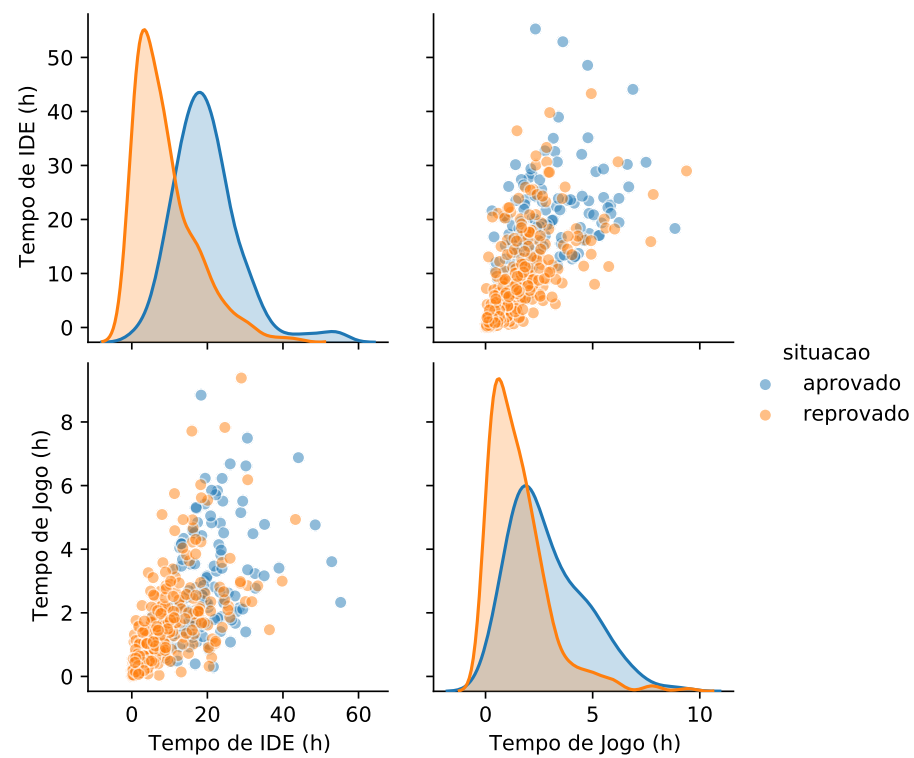

Figura 3. Comparação das distribuições das variáveis Tempo de jogo e Tempo de IDE dos alunos aprovados (em laranja) e dos alunos reprovados (em azul).

teste de Mann-Whitney (Tabela 2), pois ambas as distribuições não são normais. Com esse teste, o objetivo é saber se uma população tende a ter valores médios maiores do que a outra. Os resultados mostram que o tempo de uso do IDE e do jogo, pelos alunos que foram aprovados, são estatisticamente superiores $(p-$ value $<0,05)$ aos dos alunos reprovados, o que reforça uma relação positiva do tempo de uso desses ambientes com a aprovação dos alunos.

Tabela 2. Teste de Mann-Whitney, comparando alunos aprovados e reprovados.

\begin{tabular}{lrr}
\hline & Tempo de IDE & Tempo de Jogo \\
\hline Mann-Whitney U & 6082,000 & 9235,500 \\
\hline Z & $-11,008$ & $-8,264$ \\
\hline Tamanho de efeito $\left(\eta^{2}\right)$ & 0,29 & 0,16 \\
\hline Asymp. Sig. (bicaudal) & $1,75 \mathrm{e}-28$ & $7,06 \mathrm{e}-17$ \\
\hline
\end{tabular}

O teste de significância estatística aplicado foi bicaudal (teoria assimptótica) tendo obtido um resultado pequeno para o valor $p$, o que indica uma pequena probabilidade de erro do teste de Mann-Whitney. Porém, além do valor $p$, é importante calcular o tamanho do efeito para avaliar a magnitude da afirmação estatística. Para tal, foi utilizado o Eta quadrado $\left(\eta^{2}\right)$. Os valores obtidos (Tabela 2) são considerados altos [Cohen 2013] e, consequentemente, a magnitude da diferença entre os grupos dos estudantes aprovados e reprovados, em termos de tempo de uso do IDE e tempo de jogo, é considerada alta.

Com as fortes correlações positivas entre o tempo de uso do IDE e a nota final, aliadas à tendência dos alunos aprovados acumularem mais tempo nas duas plataformas, pode-se afirmar que os alunos que passaram mais tempo programando obtiveram maiores notas, por aprenderem através da prática. Entretanto, a correlação entre tempo de jogo e tempo de IDE se mostrou quase tão forte quanto a correlação entre tempo de IDE e nota final, o que pode indicar que alunos que praticaram mais também jogaram mais, ou vice-versa. Portanto, é possível concluir que a gamificação esteve mais presente entre os 
IX Congresso Brasileiro de Informática na Educação (CBIE 2020)

Anais do XXXI Simpósio Brasileiro de Informática na Educação (SBIE 2020)

alunos que, de certa forma, se interessaram pela disciplina.

Com isso, mostrou-se que o tempo de jogo está relacionado com o desempenho do aluno, mas está mais ligado ao tempo de prática, e este, por sua vez, leva a melhores resultados nas notas. Portanto, a hipótese $\mathbf{H 1}$ foi rejeitada, pois foram encontradas evidências que indicam que a correlação entre a nota e o tempo de jogo é forte e estatisticamente significante. Ainda, a correlação entre o tempo de jogo e o tempo de IDE foi descoberta forte e mais presente na parcela de alunos que se dedicou à disciplina. Desta forma, conclui-se que a hipótese $\mathbf{H 2}$ também foi rejeitada, já que há uma correlação forte e estatisticamente significante entre o tempo resolvendo exercícios e o tempo de jogo.

Com efeito, é possível afirmar que, neste contexto, a gamificação está relacionada de forma positiva com a prática de resolução de exercícios. Alunos que se dedicaram mais a resolver os exercícios no IDE do juiz on-line jogaram mais na plataforma de gamificação, e alunos que se interessaram pela plataforma passaram mais tempo na resolução dos exercícios no IDE.

\section{Conclusão e trabalhos futuros}

Na proporção que cresce o interesse de pesquisadores pela utilização de gamificação em ambientes educacionais, aumenta a necessidade por propostas que avaliem e mensurem os benefícios para os estudantes. Grande parte dos trabalhos examinam as ferramentas gamificadas com base em entrevistas e questionários respondidos pelos estudantes. Apesar da importância dessas propostas, elas podem causar certo viés, uma vez que o engajamento e a motivação são estados afetivos que possuem gatilhos específicos e não duram muito tempo [Pereira et al. 2020]. Uma alternativa promissora, é a utilização de dados oriundos das ações dos estudantes no sistema. Seguindo essa linha, este trabalho, analisou o desempenho dos alunos na disciplina Introdução à Programação de Computadores (IPC), considerando os aspectos comportamentais desses alunos ao utilizar a plataforma gamificada CodePlay, integrada ao juiz on-line CodeBench.

Foi realizado um estudo com dados de 9 turmas de IPC, da Universidade Federal do Amazonas, coletados no primeiro semestre de 2019. As análises mostraram que há uma correlação forte e estatisticamente significante entre o tempo de resolução de exercícios e o tempo de permanência no jogo. Além disso, o tempo de uso da plataforma de gamificação e do IDE possuem correlação forte e positiva com o desempenho dos alunos. Em todos os casos analisados, as correlações são estatisticamente significantes ( $p$-value $<0.05$ ). Desta forma, foi evidenciado que a gamificação está positivamente relacionada à prática de resolução de exercícios, uma vez que, os alunos com maior dedicação à resolução dos exercícios no juiz on-line também utilizaram mais a plataforma gamificada, e vice-versa. Portanto, mostrou-se que a gamificação possui forte correlação com o bom desempenho dos alunos, principalmente quanto ao tempo de prática.

Como trabalhos futuros, pretende-se avaliar o comportamento em cada uma das 9 turmas, de forma a verificar se os cursos têm perfis diferentes de alunos, para, então estudar novas formas de utilização e avaliação da gamificação. Além disso, pretende-se utilizar o ambiente gamificado em outros períodos e instituições de ensino para a aumentar a quantidade e diversidade dos dados.

\section{Agradecimentos}

Esta pesquisa, realizada no âmbito do Projeto Samsung-UFAM de Ensino e Pesquisa (SUPER), nos termos do artigo 48 do Decreto $n^{\circ}$ 6.008/2006 (SUFRAMA), foi parcialmente 
IX Congresso Brasileiro de Informática na Educação (CBIE 2020)

Anais do XXXI Simpósio Brasileiro de Informática na Educação (SBIE 2020)

financiada pela Samsung Eletrônica da Amazônia Ltda., Nos termos da Lei Federal $\mathrm{n}^{\mathrm{o}}$ 8.387/1991, por meio dos convênios 001/2020 e 003/2019, firmados com a Universidade Federal do Amazonas e a FAEPI, Brasil. O presente trabalho foi realizado com apoio da Coordenação de Aperfeiçoamento de Pessoal de Nível Superior - Brasil (CAPES) Código de Financiamento 001.

\section{Referências}

Cohen, J. (2013). Statistical power analysis for the behavioral sciences. Academic press.

Dichev, C. and Dicheva, D. (2017). Gamifying education: what is known, what is believed and what remains uncertain: a critical review. International journal of educational technology in higher education, 14(1):9.

Dwan, F., Oliveira, E., and Fernandes, D. (2017). Predição de zona de aprendizagem de alunos de introdução à programação em ambientes de correção automática de código. In Brazilian Symposium on Computers in Education (Simpósio Brasileiro de Informática na Educação-SBIE), volume 28, page 1507.

Klock, A. C. T., Ogawa, A. N., Gasparini, I., and Pimenta, M. S. (2018). Does gamification matter?: a systematic mapping about the evaluation of gamification in educational environments. In Proceedings of the 33rd Annual ACM Symposium on Applied Computing, pages 2006-2012. ACM.

Meder, M., Plumbaum, T., and Albayrak, S. (2017). A primer on data-driven gamification design. In DDGD@MindTrek, pages 12-17.

Ogawa, A., Klock, A. C. T., and Gasparini, I. (2016). Avaliação da gamificação na área educacional: um mapeamento sistemático. In Brazilian Symposium on Computers in Education (Simpósio Brasileiro de Informática na Educação-SBIE), volume 27, page 440 .

Pereira, F. D., Oliveira, E., Cristea, A., Fernandes, D., Silva, L., Aguiar, G., Alamri, A., and Alshehri, M. (2019). Early dropout prediction for programming courses supported by online judges. In International Conference on Artificial Intelligence in Education, pages 67-72. Springer.

Pereira, F. D., Toda, A., Oliveira, E. H., Cristea, A. I., Isotani, S., Laranjeira, D., Almeida, A., and Mendonça, J. (2020). Can we use gamification to predict students' performance? a case study supported by an online judge. In International Conference on Intelligent Tutoring Systems, pages 259-269. Springer.

Pessoa, M., Fernandes, D., de Carvalho, L. S. G., Oliveira, E., Nakamura, W., and Conte, T. (2019). Codeplay: Uma plataforma de gamificação baseada em jogos de rpg multiplayer. In Brazilian Symposium on Computers in Education (Simpósio Brasileiro de Informática na Educação-SBIE), volume 30, page 843.

Sailer, M. and Homner, L. (2020). The gamification of learning: A meta-analysis. Educational Psychology Review, pages 77-112.

Stuart, H., Lavoué, E., and Serna, A. (2020). To tailor or not to tailor gamification? an analysis of the impact of tailored game elements on learners' behaviours and motivation. In 21th International Conference on Artificial Intelligence in Education.

Toda, A. M., Valle, P. H., and Isotani, S. (2017). The dark side of gamification: An overview of negative effects of gamification in education. In Researcher Links Workshop: Higher Education for All, pages 143-156. Springer. 